\title{
Edge-preserving sectional image reconstruction in optical scanning holography
}

\author{
Xin Zhang and Edmund Y. Lam* \\ Imaging Systems Laboratory, Department of Electrical and Electronic Engineering, University of Hong Kong, \\ Pokfulam Road, Hong Kong, China \\ *Corresponding author: elam@eee.hku.hk
}

Received March 25, 2010; revised May 19, 2010; accepted May 21, 2010; posted May 21, 2010 (Doc. ID 126004); published June 16, 2010

\begin{abstract}
Optical scanning holography (OSH) enables us to capture the three-dimensional information of an object, and a post-processing step known as sectional image reconstruction allows us to view its two-dimensional crosssection. Previous methods often produce reconstructed images that have blurry edges. In this paper, we argue that the hologram's two-dimensional Fourier transform maps into a semi-spherical surface in the threedimensional frequency domain of the object, a relationship akin to the Fourier diffraction theorem used in diffraction tomography. Thus, the sectional image reconstruction task is an ill-posed inverse problem, and here we make use of the total variation regularization with a nonnegative constraint and solve it with a gradient projection algorithm. Both simulated and experimental holograms are used to verify that edge-preserving reconstruction is achieved, and the axial distance between sections is reduced compared with previous regularization methods. (C) 2010 Optical Society of America
\end{abstract}

OCIS codes: $090.1760,090.1995,100.3020,100.3190,180.6900,110.1758,170.3880$.

\section{INTRODUCTION}

Optical scanning holography $(\mathrm{OSH})$ is a digital holographic technique that preserves phase information by optical heterodyne detection and records the threedimensional (3D) diffracted propagation of an object as two-dimensional (2D) holograms [1,2]. Computational methods are then employed to reconstruct individual $2 \mathrm{D}$ sectional images, and recent techniques aim to enhance the quality of reconstructed sections with higher resolution [3,4]. This includes an inverse imaging method, where the $\mathrm{OSH}$ system is modeled by an operation matrix and sectional image reconstruction is cast as an ill-posed inverse problem [5]. Tikhonov regularization is then used as a smoothness constraint. While this algorithm has shown a very good reconstruction quality in terms of suppressing defocus noise compared with other recent methods [6,7], sharp edges tend to be blurred as a result of minimizing the total energy (or mean square error) in the reconstruction algorithm [8].

It may seem straightforward, then, to experiment with replacing the Tikhonov regularization by other nonlinear techniques. However, we argue that one should first understand the imaging process and investigate how the data are undersampled. This can help us appreciate how prior information can be needed, and how it may be formulated in the nonlinear regularization. As we will see in the third section, with encoding the $3 \mathrm{D}$ information of an object into a $2 \mathrm{D}$ hologram by optical coherent detection [9], OSH effectively captures an object's diffracted propagation. Therefore, the sectional image reconstruction is equivalent to retrieving sections from the diffraction, which is known as diffraction tomography in a variety of applications, ranging from ultrasound imaging to geophysical exploration [10,11]. Proposed algorithms have evolved from interpolation based on Born and Rytov approximations to filtered backpropagation (FBP) [12], and to inverse scattering algorithms and total variation (TV) minimization more recently [13,14]. The amount of data capture is gradually decreasing with more advanced algorithms. Thus, in relating OSH to diffraction tomography, we can take advantage of these more recent techniques in reconstructing sectional images.

This paper is organized as follows. Section 2 describes the OSH system. Then, in Section 3, we analyze the relationship between diffraction tomography and the $\mathrm{OSH}$ system. A sampling of 3D Fourier transform result is presented in the mechanism of generating the hologram. Section 4 presents the inverse problem of representing a reconstruction of sectional images from a hologram. Both the total variation (TV) penalty and the nonnegativity are used as constraints to regularize a solution. In Section 5, experimental results are presented to demonstrate the performance of the reconstruction method. Section 6 concludes the paper.

\section{OPTICAL SCANNING HOLOGRAPHY SYSTEM}

In OSH, an object is scanned by a system with a complex Fresnel-zone-plate (FZP) impulse response [15]. Then heterodyne detection collects diffracted and scattered light from the object and records it as electronic holograms. The holograms encode the $3 \mathrm{D}$ position information of the diffracted propagation through the object.

Fig. 1 shows a schematic diagram of an OSH system. A helium-neon (HeNe) laser is used to scan an object. A beam splitter $\mathrm{BS}_{1}$ splits the laser beam. One beam works 


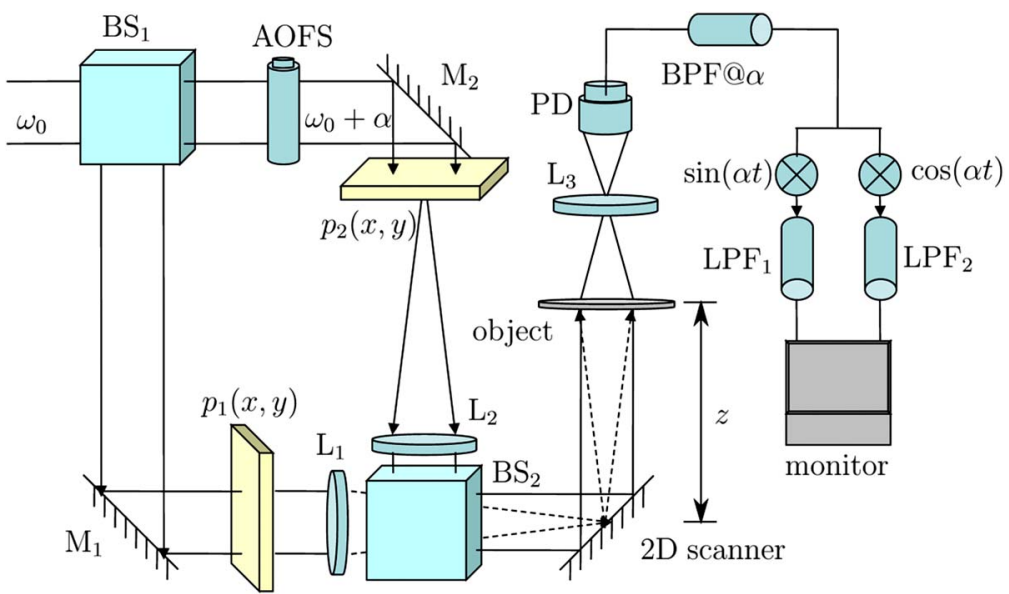

Fig. 1. (Color online) Schematic diagram of the optical scanning holography system.

as a reference light, and the other is an object light to scan the object. The former goes through an acousto-optic frequency shifter (AOFS) to alter its temporal frequency from $\omega_{0}$ to $\omega_{0}+\alpha$, which is then reflected by a mirror $\mathrm{M}_{2}$ to illuminate a pupil, $p_{2}(x, y)$. Meanwhile, the object light is reflected by $\mathrm{M}_{1}$ and controlled by another pupil, $p_{1}(x, y)$. The two controlled light beams go through lenses $\mathrm{L}_{2}$ and $\mathrm{L}_{1}$, respectively, and are then combined by another beam splitter $\mathrm{BS}_{2}$. The combined light is used by a $2 \mathrm{D}$ scanner to scan an object. A photodetector PD accepts all light from the object and sends an electric signal to the demodulation part, which includes a band-pass filter BPF tuned at the frequency $\alpha$, electronic multipliers, and lowpass filters LPFs. Finally, quadrature electronic holograms containing the complete information of the object are recorded $[16,17]$. Holograms we measure by the OSH system are complex and we often separate them into real and imaginary parts in viewing.

The optical transfer function (OTF) in $\mathrm{OSH}$ is given by

$$
H\left(k_{x}, k_{y} ; z\right)=\exp \left\{-\frac{j z}{2 k_{0}}\left(k_{x}^{2}+k_{y}^{2}\right)\right\}
$$

where $z$ is the distance between the scanner and a particular section of interest of the object, $k_{0}$ is the wavenumber, and $k_{x}$ and $k_{y}$ are the transverse spatial frequency coordinates. Derivations of this formula can be found in $[16,17]$.

\section{OSH AS DIFFRACTION TOMOGRAPHY}

\section{A. OSH in Fourier Basis}

To express the OSH data capture using Fourier bases, we first note that the wavenumber $k_{0}$ is related to $k_{x}, k_{y}$, and $k_{z}$, which are the $x, y$, and $z$ components of the propagation vector, by $k_{0}=\sqrt{k_{x}^{2}+k_{y}^{2}+k_{z}^{2}}$. Making use of the paraxial approximation [18], we have

$$
\begin{aligned}
k_{z}= & \left(k_{0}^{2}-k_{x}^{2}-k_{y}^{2}\right)^{1 / 2}=k_{0}\left(1-\frac{k_{x}^{2}+k_{y}^{2}}{k_{0}^{2}}\right)^{1 / 2} \\
\approx & k_{0}\left(1-\frac{1 k_{x}^{2}+k_{y}^{2}}{2}\right), \\
& \text { provided } k_{x}^{2}+k_{y}^{2} \ll k_{0}^{2}=k_{0}-\frac{k_{x}^{2}+k_{y}^{2}}{2 k_{0}} .
\end{aligned}
$$

The requirement of $k_{x}^{2}+k_{y}^{2} \ll k_{0}^{2}$ is reasonable in OSH because we assume that waves are forward propagating along the positive $z$ direction. Thus, the $x$ and $y$ components are much smaller in comparison.

Substituting the approximation into Eq. (1), we can express the OTF as [19]

$$
H\left(k_{x}, k_{y} ; z\right)=\exp \left\{-\frac{j z}{2 k_{0}}\left(k_{x}^{2}+k_{y}^{2}\right)\right\} \approx \exp \left\{-j z\left(k_{0}-k_{z}\right)\right\} .
$$

Now consider an object $g(x, y ; z)$ with the Fourier transform of its squared intensity $G\left(k_{x}, k_{y} ; z\right)=\mathcal{F}_{x y}\left\{|g(x, y ; z)|^{2}\right\}$. The resulting hologram, denoted as $q(x, y)$, is given by

$$
q(x, y)=\int_{-\infty}^{\infty} \mathcal{F}_{x y}^{-1}\left\{G\left(k_{x}, k_{y} ; z\right) H\left(k_{x}, k_{y} ; z\right)\right\} \mathrm{d} z .
$$

Making use of the approximation in Eq. (3), we get

$$
\begin{aligned}
q(x, y) \approx & \iint_{-\infty}^{\infty} \int_{x} G\left(k_{x}, k_{y} ; z\right) \exp \left\{-j z\left(k_{0}-k_{z}\right)\right\} \\
& \times \exp \left\{j\left(x k_{x}+y k_{y}\right)\right\} \mathrm{d} k_{x} \mathrm{~d} k_{y} \mathrm{~d} z \\
= & \iint_{-\infty}^{\infty}\left(G\left(k_{x}, k_{y} ; z\right) \exp \left\{-j z k_{0}\right\}\right) \\
& \times \exp \left\{j\left(x k_{x}+y k_{y}+k_{z} z\right)\right\} \mathrm{d} k_{x} \mathrm{~d} k_{y} \mathrm{~d} z .
\end{aligned}
$$

In words, this states that the hologram from OSH equals the $3 \mathrm{D}$ inverse Fourier transform of the 2D Fourier trans- 
form of (the squared intensity of) the object multiplied by a linear phase factor.

\section{B. Fourier Diffraction Theorem in OSH}

A simpler statement can in fact be made if we consider, instead, the Fourier transform of the hologram, which we denote as $Q\left(k_{x}, k_{y}\right)$. From Eq. (4), we have

$$
\begin{aligned}
Q\left(k_{x}, k_{y}\right)= & \int_{-\infty}^{\infty} G\left(k_{x}, k_{y} ; z\right) H\left(k_{x}, k_{y} ; z\right) \mathrm{d} z \\
\approx & \int_{-\infty}^{\infty} \mathcal{F}_{x y}\left\{|g(x, y ; z)|^{2}\right\} \exp \left\{j z\left(k_{z}-k_{0}\right)\right\} \mathrm{d} z \\
= & \iint_{-\infty}^{\infty} \int|g(x, y ; z)|^{2} \exp \left\{-j\left[x k_{x}+y k_{y}-z\left(k_{z}\right.\right.\right. \\
& \left.\left.\left.-k_{0}\right)\right]\right\} \mathrm{d} x \mathrm{~d} y \mathrm{~d} z .
\end{aligned}
$$

Letting $\widetilde{G}\left(\widetilde{k}_{x}, \widetilde{k}_{y}, \widetilde{k}_{z}\right)=\mathcal{F}_{x y z}\left\{|g(x, y ; z)|^{2}\right\}$, i.e., the 3D Fourier transform of the squared magnitude of the object, we can further simplify $Q\left(k_{x}, k_{y}\right)$ as

$Q\left(k_{x}, k_{y}\right)=\widetilde{G}\left(k_{x}, k_{y},-\left(k_{z}-k_{0}\right)\right)=\widetilde{G}\left(k_{x}, k_{y}, k_{0}-\sqrt{k_{0}^{2}-k_{x}^{2}-k_{y}^{2}}\right)$.

In words, the samples of $Q\left(k_{x}, k_{y}\right)$ comprise points equidistant from the point $\left(0,0, k_{0}\right)$ in the $3 \mathrm{D}$ spatial frequency domain. This is a semi-spherical surface depicted in Fig. 2.

We can interpret the relationship in Eq. (7) in light of the Fourier diffraction theorem commonly used in diffraction tomography. OSH records a diffracted propagation through the object by a collimated light. The theorem states that when an object is illuminated by a plane wave, the Fourier transform of the diffracted field produces the Fourier transform of the object along the semi-spherical surface in the 3D spatial frequency domain [20,21]. In a sense, this is a generalization of the projection-slice theorem [22], applicable to cases such as computed tomography (CT), by taking into account the wave nature of light propagation and therefore incorporating the diffraction effect [23]. The acquired data also form a "slice" in the spatial frequency domain, albeit the slice is bent to a semi-spherical surface.

Our interest is in the reconstruction of $|g(x, y ; z)|^{2}$ from the acquired slice. In CT, multiple slices are acquired, and methods such as filtered back-projection can be employed to reconstruct the volumetric data [24]. This is analytically "perfect" in the absence of noise. On the other hand, in OSH we effectively receive only a single semi-spherical slice of data in the spatial frequency domain. The reconstruction problem is therefore severely underdetermined, which explains why regularization is necessary to cope with its ill-posed nature [5]. In addition, many recent algorithms in the reconstruction of CT and other signals involve making use of prior information of the object, particularly the sparsity of its gradient $[25,26]$, to ensure that the reconstructed image contains sharp boundaries. We make use of this to produce our edge-preserving $\mathrm{OSH}$ sectional image reconstruction, as detailed in Section 4.

\section{EDGE-PRESERVING RECONSTRUCTION}

We develop our edge-preserving reconstruction in the spatial domain as follows. Referring back to Eq. (4), we can express the capturing of the hologram as

$$
q(x, y)=\int_{-\infty}^{\infty}|g(x, y ; z)|^{2} * h(x, y ; z) \mathrm{d} z
$$

where $\quad h(x, y ; z)=\mathcal{F}_{x y}^{-1}\left\{H\left(k_{x}, k_{y} ; z\right)\right\}=-j k_{0} / 2 \pi z \exp \left\{j k_{0}\left(x^{2}\right.\right.$ $\left.\left.+y^{2}\right) / 2 z\right\}$ as derived in $[5,17]$. We then discretize $z$ as $z_{1}, z_{2}, \ldots z_{n}$, and represent the lexicographical ordering of $q(x, y)$ and $\left|g\left(x, y ; z_{i}\right)\right|^{2}$ by $\mathbf{q}$ and $\mathbf{g}_{i}$, respectively. The convolution operation is written as a matrix-vector multiplication by forming the matrix $\mathbf{H}_{i}$ from $h\left(x, y ; z_{i}\right)$ [27], so that Eq. (8) becomes

$$
\mathbf{q}=\sum_{i=1}^{n} \mathbf{H}_{i} \mathbf{g}_{i}=\mathbf{H g},
$$

where $\mathbf{H}=\left[\mathbf{H}_{1} \cdots \mathbf{H}_{n}\right]$ and $\mathbf{g}=\left[\mathbf{g}_{1}^{T} \cdots \mathbf{g}_{n}^{T}\right]^{T}$. Our sectional image reconstruction problem is then an inverse problem, where we seek to estimate $\mathbf{g}$ from the observation $\mathbf{q}$ and a known $\mathbf{H}$.

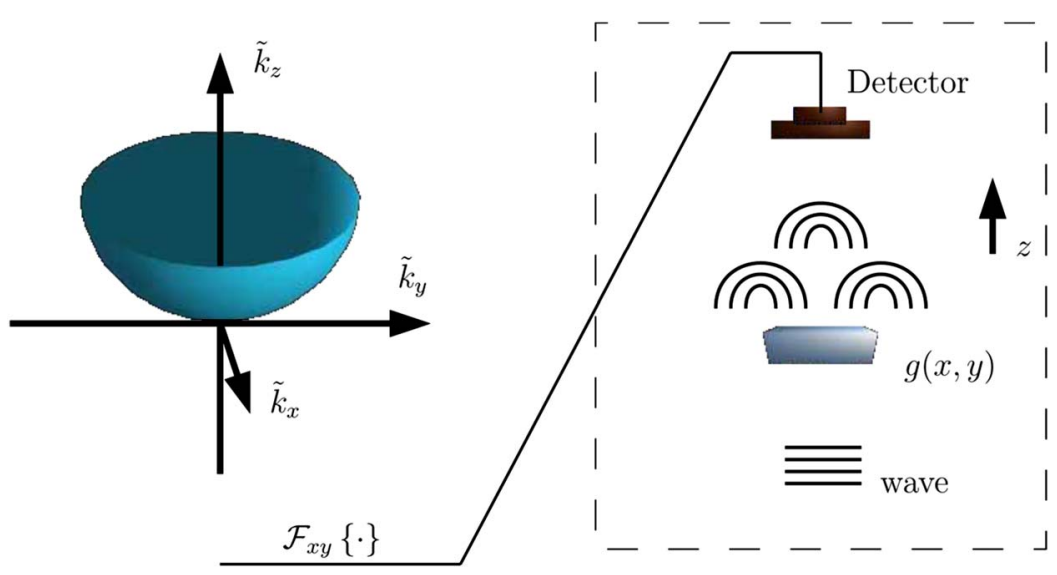

Fig. 2. (Color online) 2D slice of the 2D Fourier transform of a diffraction in the spatial frequency domain. 


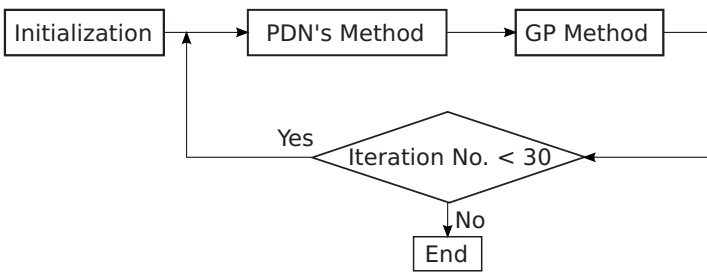

Fig. 3. Flowchart of edge-preserving reconstruction method ("PDN's Method" stands for the primal-dual Newton's method and "GP Method" stands for the gradient projection method).

To solve this, one can formulate a regularized least squares problem by minimizing the function

$$
J(\mathbf{g})=\|\mathbf{q}-\mathbf{H g}\|^{2}+\alpha\|\mathbf{C g}\|^{2},
$$

where the $\mathbf{C}$ matrix is chosen to be a high-pass filter, such as Laplacian of Gaussian, and $\alpha$ is the regularization parameter, which balances between smoothness and fidelity to observed data. The $\ell_{2}$ norm is used in both instances in this equation. This is the Tikhonov regularization algorithm developed in [5]. Note that in this method, smoothness is imposed on the whole reconstructed image at the expense of reconstructing sharp edges. Hence, although the reconstruction quality and the flexibility of this algorithm show advantage over other recent schemes [6,7], one noticeable disadvantage of this algorithm is that the reconstructed objects often show blurred edges. This not only affects the visual appearance, but also presents challenges in possible further image analysis algorithms, such as identifying the size of an object.

To improve on the edge quality, we can instead minimize the function

$$
J_{\mathrm{TV}}(\mathbf{g})=\|\mathbf{q}-\mathbf{H g}\|^{2}+\alpha\|\mathbf{g}\|_{\mathrm{TV}},
$$

where $\|\cdot\|_{\mathrm{TV}}$ stands for the total variation (TV) regularization given by

$$
\|v(x, y)\|_{\mathrm{TV}}=\int_{-\infty}^{\infty} \int_{-\infty}|\nabla v(x, y)| \mathrm{d} x \mathrm{~d} y .
$$

In addition, $\nabla v(x, y)=(\partial v / \partial x, \partial v / \partial y)$ stands for the gradient of $v(x, y)$.

Such TV regularization has shown to be capable of maintaining edge sharpness in various image and video restoration problems (for example, in [28-30]). TV norm is also essentially $\ell_{1}$ norm on the gradient of an image, and it has been shown in recent years that this is a powerful tool to reconstruct images from very limited data if we can assume sparsity in signal transition (i.e., edges) in an image [31,32]. In our OSH sectional image reconstruction problem, we indeed have only very limited data as pointed out in Section 3. To the best of our knowledge, the present paper is the first report on a TV regularizationbased sectional image reconstruction algorithm to tackle this problem.

Moreover, the computational of $\mathbf{H g}$ also differentiates the present problem from other image restoration algorithms. It does not represent a convolution, but a sum of convolutions; as such, we cannot directly apply the fast Fourier transform to implement the matrix-vector product efficiently. Instead, we developed a method earlier (which is reported in [33]) for this specific purpose. In addition, a further refinement to Eq. (4) is also possible. Since $\mathbf{g}_{i}$ contains entries in $\left|g\left(x, y ; z_{i}\right)\right|^{2}$, it must be nonnegative for all pixels. Thus, we should seek to minimize

$$
\hat{J}_{\mathrm{TV}}(\mathbf{g})=\|\mathbf{q}-\mathbf{H g}\|^{2}+\alpha\|\mathbf{g}\|_{\mathrm{TV}}, \quad \text { subject to } \mathbf{g} \geqslant 0,
$$

where the inequality is taken to be componentwise. To solve this, we can make use of the primal-dual Newton's method [34] and combine it with a gradient projection method to regularize the solution. In each iteration, the primal-dual Newton's method gives a preliminary guess of the solution, which should be regularized by the TV penalty. However, the solution may be negative in certain entries, which does not agree with the nonnegative intensity captured by the scanning holography system [35] . This is fixed by the gradient projection method, which updates the solution to make it nonnegative. A flowchart of the reconstruction is shown in Fig. 3. Here the regularization parameter is not very sensitive, and we set it to 100 in general. Iterating these steps allows us to obtain a solution that satisfies the TV regularization and nonnegativity at the same time.

\section{EXPERIMENTS}

An experiment is conducted to demonstrate the performance of this edge-preserving reconstruction algorithm. To be consistent with previous work in order to allow convenient comparison, we use a set of simulated data that include two distinct sections in the $z$ direction, where each section contains a rectangular bar. The two bars are at $z_{1}=14 \mathrm{~mm}$ and $z_{2}=15 \mathrm{~mm}$ away from the scanner, as

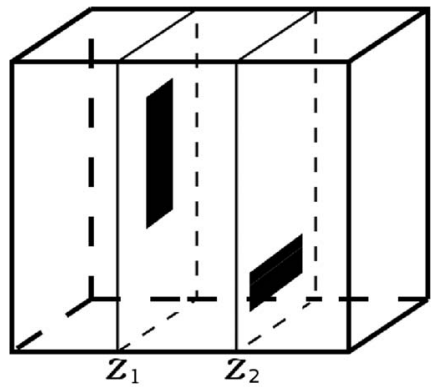

(a)

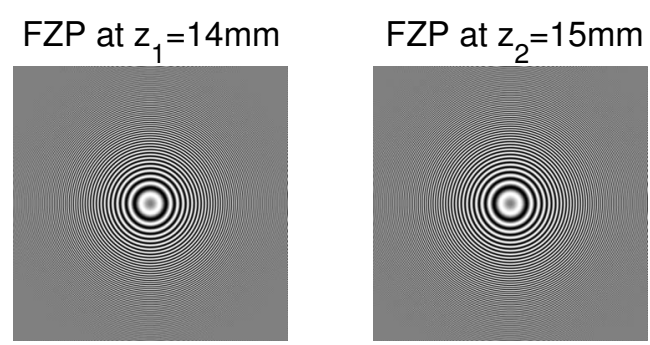

(b)

Fig. 4. Object and FZPs in the first experiment. Shown in (a) is the object, in which two elements (i.e., rectangular bars) are at $z_{1}$ and $z_{2}$ sections. Shown in (b) are the real parts of FZPs of a point source at $z_{1}$ and $z_{2}$. 


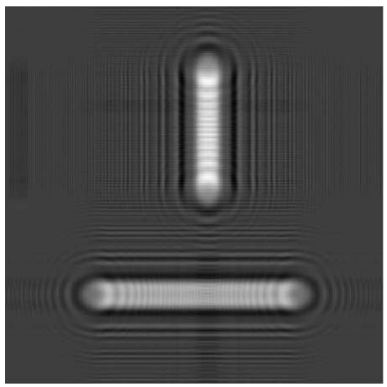

(a)

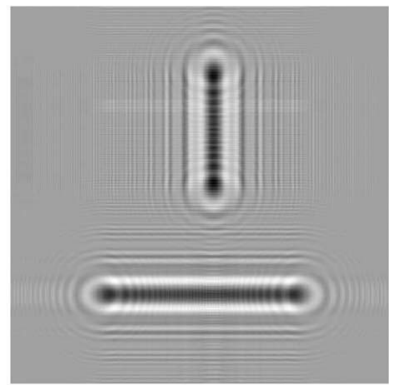

(b)

Fig. 5. Holograms containing two-sectional images of the object in the first experiment. Sine hologram in (a) is the real part and cosine hologram in (b) is the imaginary part.

shown in Fig. 4(a). The object is then imported into Eq. (8) to generate a simulated hologram for the experiment. Shown in Fig. 4(b) are the real parts of the Fresnel zone plates [16] of a point source at $z_{1}$ and $z_{2}$, respectively. The real and imaginary parts of the hologram are shown in Fig. 5, where (a) is the sine hologram (the real part) and (b) is the cosine hologram (the imaginary part).

The reconstruction of the two sections from the hologram is shown in Fig. 6. As can be seen, the two sections are well separated and are reconstructed at the correct location and with sharp edges. Defocus noise, which is a problem with conventional techniques [16], does not have any visible effect on the reconstructed sections. Moreover, we can compare this reconstruction with the one using the Tikhonov regularization, as shown in Fig. 7. With this method, the two reconstructed sections also show the corresponding bars clearly, but with more defocus noise. The intensity in each section is also recovered nonuniformly, i.e., corners and edges are recovered with higher intensity, while the middle parts have lower intensity in comparison.

To look into the quality of edge preservations of our proposed method, we extract a cross-section of the middle row of the section at $z_{1}$, which is shown in Fig. 8 . The blue dashed line shows the row in the original object, while the red solid curve is from the reconstructed section. We can observe that the two agree quite well. In particular, while the ideal object exhibits an abrupt change at the edge within one pixel, in our reconstruction the change can take place within two pixels. Another region of notice is the flat area with high intensity values, which corresponds to the area within the object. In our reconstruc-

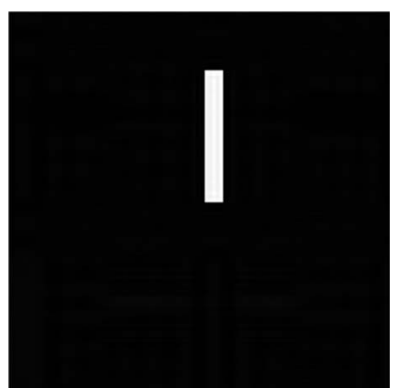

(a)

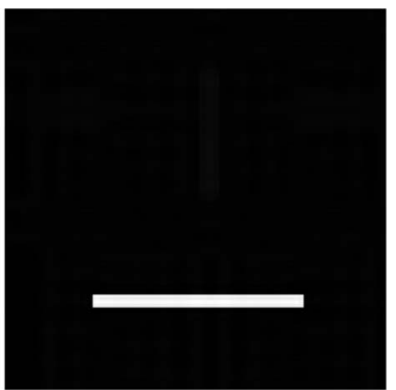

(b)
Fig. 6. Reconstructed sections by the TV regularization method. (a) Section at $z_{1}=14 \mathrm{~mm}$ and (b) at $z_{2}=15 \mathrm{~mm}$.

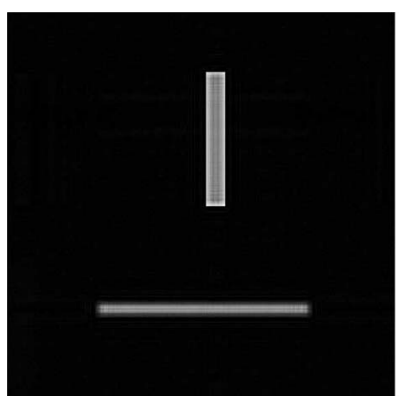

(a)

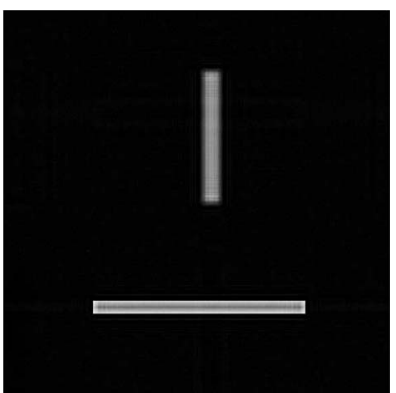

(b)
Fig. 7. Reconstructed sections by the Tikhonov regularization method. (a) Section at $z_{1}=14 \mathrm{~mm}$ and (b) at $z_{2}=15 \mathrm{~mm}$.

tion, the intensities are not flat, but contain no more than $5 \%$ variations. This gives rise to a rather uniform visual brightness of the object.

Another comparison between the edge-preserving reconstruction and the Tikhonov-regularized inverse imaging methods is the axial distance $\Delta z$ between the two bars needed for the reconstruction to show two distinct objects at different depths. In this experiment, the hologram is of size $2 \mathrm{~mm}$ by $2 \mathrm{~mm}$, which translates to $500 \times 500$ pixels. The lateral resolution of the hologram is then given by $\Delta x=\Delta y=4 \mu \mathrm{m}$. As shown in Fig. 6, the edge-preserving reconstruction separates the two sections with $\Delta z=1 \mathrm{~mm}$ completely, while the reconstruction with Tikhonov regularization fails to separate them while suppressing defocus noise, as shown in Fig. 7. (Note that in [5], Tikhonov regularization was successfully applied to separate two bars with $\Delta z=1 \mathrm{~mm}$, but in that experiment $\Delta x=\Delta y$ $=1 \mu \mathrm{m}$, which translates to greater constraints if we were to realize this in a physical system.) If the section at $z_{2}$ is moved to $24 \mathrm{~mm}$ and a new hologram is measured, the reconstruction by Tikhonov regularization shows comparable performance as our method here in terms of defocus noise suppression. Thus, it can be argued that with the

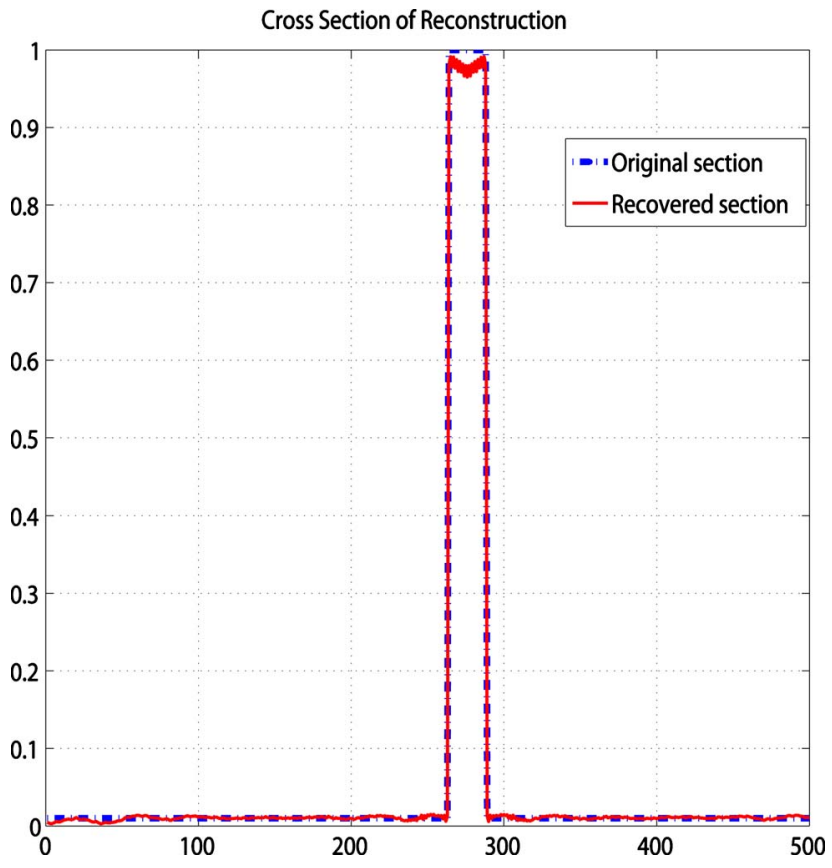

Fig. 8. (Color online) Cross-section of the middle row at $z_{1}$. 


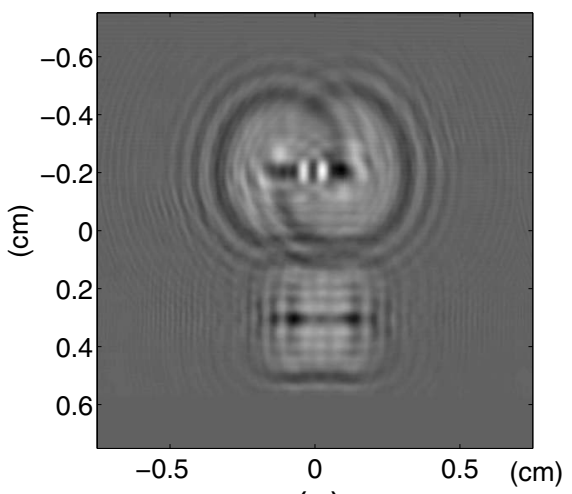

(a)

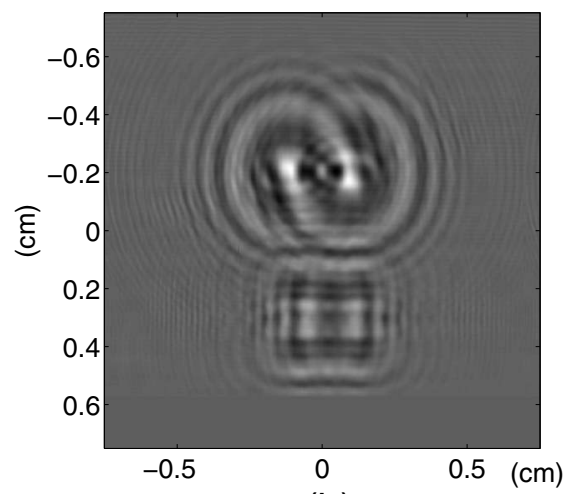

(b)

Fig. 9. Holograms measured by a physical OSH system [36]. (a) Real part of the recorded hologram. (b) Imaginary part of the recorded hologram.

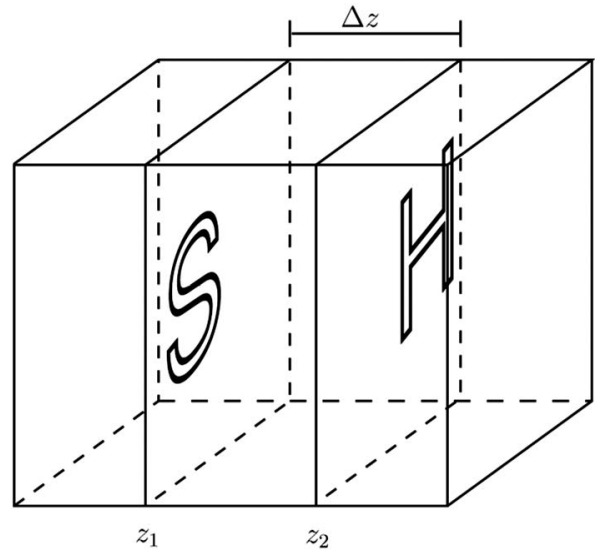

Fig. 10. Object in the second experiment scanned by a physical system.

same lateral resolution of a hologram, the edgepreserving reconstruction is more competent in separating close sections, by reducing the axial separation from $10 \mathrm{~mm}$ to $1 \mathrm{~mm}$ in this experiment.

Finally, we evaluate the current algorithm using experimental data that have been used earlier in [36]. The hologram is of size $1.5 \mathrm{~cm}$ by $1.5 \mathrm{~cm}$, and the two sections are at $z_{1}=87 \mathrm{~cm}$ and $z_{2}=107 \mathrm{~cm}$. Note that this system is $\approx 100$ times larger than that used in the previous simulations. The diameter of the collimated beam is $c=25 \mathrm{~mm}$ and the focal length of the lens is $f=500 \mathrm{~mm}$. Thus, the numerical aperture (NA) of the recorded full-parallax complex hologram is $\mathrm{NA} \asymp c / 2 f=0.025$. Shown in Fig. 9 are the real and imaginary parts of the complex hologram, where the object consists of two transparencies with the letters "S" at $z_{1}$ and "H" at $z_{2}$, as shown in Fig. 10. Note that the two letters are hollow inside, which is unlike the two solid bars discussed above, and therefore the reconstruction should preserve such hollow appearance. Our proposed reconstruction method is applied to the hologram, and the extracted sectional images are shown in Fig. 11, where (a) presents the reconstructed section at $z_{1}$ and (b) is the section at $z_{2}$. In both cases, the letters are recovered and the edges are well preserved. We compare the reconstruction with that using Tikhonov regularization, as shown in Fig. 12. Although the intensity of the recovered images is larger, this actually deviates more from the object, which consists of letters with outlines only.

\section{CONCLUSION}

In this paper we have analyzed the data collection in $\mathrm{OSH}$ and developed an edge-preserving sectional image reconstruction method that improves upon the inverse imaging method using Tikhonov regularization in an earlier work [5]. The new technique makes use of total-variation regularization and a nonnegativity constraint, and is imple-

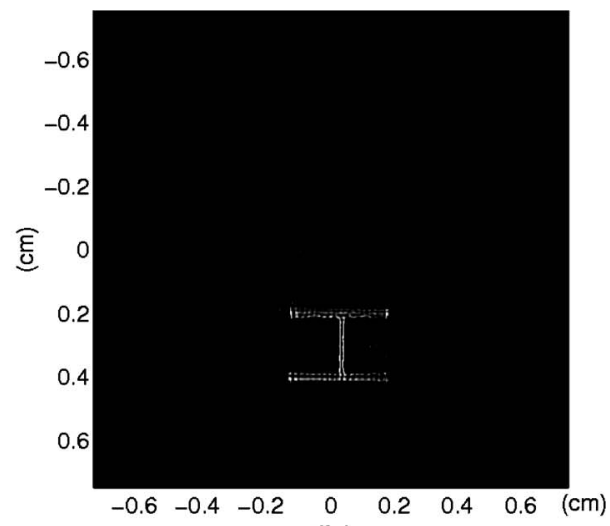

(b)

Fig. 11. Reconstructed sectional images by TV regularization and nonnegative constraint are shown in (a) and (b). 


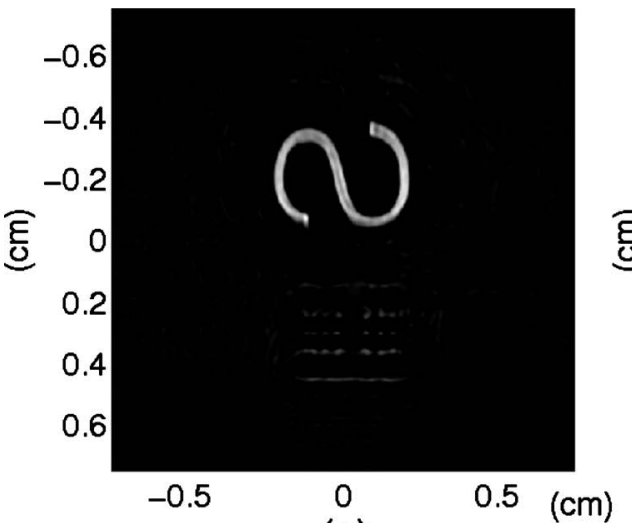

(a)

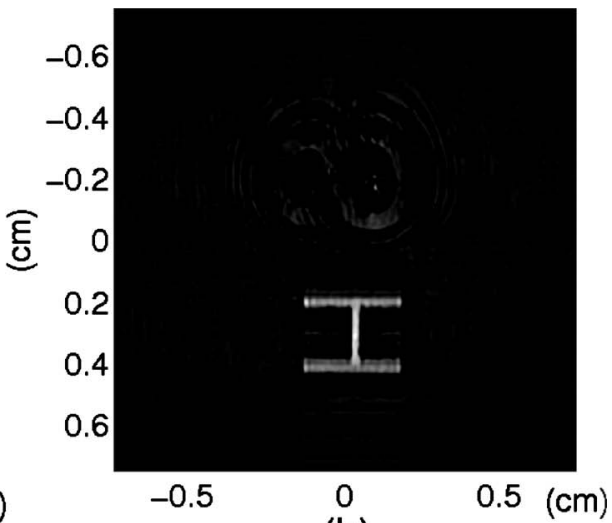

(b)

Fig. 12. Reconstructed sectional images by inverse imaging using Tikhonov regularization are shown in (a) and (b).

mented using a primal-dual Newton's method combined with gradient projection. Simulation results show good reconstruction quality with sharp edges and fairly uniform intensities within the object.

\section{ACKNOWLEDGMENTS}

The authors wish to express their appreciation for helpful discussions with Dr. Kerkil Choi regarding compressive holograms, with Prof. Terence Tao about the Fourier measurements in compressive sensing, and with Prof. Taegeun Kim about the resolution of the experimental OSH system. This work was supported in part by the University Research Committee of the University of Hong Kong under Projects 10208291, 10208648, and10400399.

\section{REFERENCES}

1. T.-C. Poon, "Scanning holography and two-dimensional image processing by acousto-optic two-pupil synthesis," J. Opt. Soc. Am. A 2, 521-527 (1985).

2. J. Swoger, M. Martínez-Corral, J. Huisken, and E. Stelzer, "Optical scanning holography as a technique for highresolution three-dimensional biological microscopy," J. Opt. Soc. Am. A 19, 1910-1918 (2002).

3. U. Schnars and W. Jueptner, Digital Holography: Digital Hologram Recording, Numerical Reconstruction, and Related Techniques (Springer-Verlag, 2005).

4. T.-C. Poon, Digital Holography and Three-Dimensional Display: Principles and Applications (Springer, 2006).

5. X. Zhang, E. Y. Lam, and T.-C. Poon, "Reconstruction of sectional images in holography using inverse imaging," Opt. Express 16, 17215-17226 (2008).

6. T. Kim, "Optical sectioning by optical scanning holography and a Wiener filter," Appl. Opt. 45, 872-879 (2006).

7. H. Kim, S.-W. Min, B. Lee, and T.-C. Poon, "Optical sectioning for optical scanning holography using phase-space filtering with Wigner distribution functions," Appl. Opt. 47, D164-D175 (2008)

8. S.-J. Kim, K. Koh, M. Lustig, S. Boyd, and D. Gorinevsky, "An interior-point method for large-scale $l_{1}$-regularized least squares," IEEE J. Sel. Top. Signal Process. 1, 606-617 (2007).

9. G. Indebetouw, "Properties of a scanning holographic microscopy: Improved resolution, extended depth-of-focus, and/or optical sectioning," J. Mod. Opt. 49, 1479-1500 (2002).

10. R. K. Mueller, M. Kaveh, and G. Wade, "Reconstructive tomography and applications to ultrasonics," Proc. IEEE 67, 567-587 (1979).
11. A. J. Devaney, "Geophysical diffraction tomography," IEEE Trans. Geosci. Remote Sens. GE-22, 3-13 (1984).

12. A. J. Devaney, "A filtered backpropagation algorithm for diffraction tomography," Ultrason. Imaging 4, 336-350 (1982).

13. P. Guo and A. J. Devaney, "Comparison of reconstruction algorithms for optical diffraction tomography," J. Opt. Soc. Am. A 22, 2338-2347 (2005).

14. S. J. LaRoque, E. Y. Sidky, and X. Pan, "Accurate image reconstruction from few-view and limited-angle data in diffraction tomography," J. Opt. Soc. Am. A 25, 1772-1782 (2008).

15. G. Indebetouw, P. Klysubun, T. Kim, and T.-C. Poon, "Imaging properties of scanning holographic microscopy," J. Opt. Soc. Am. A 17, 380-390 (2000).

16. T.-C. Poon, Optical Scanning Holography with MATLAB (Springer-Verlag, 2007).

17. E. Y. Lam, X. Zhang, H. Vo, T.-C. Poon, and G. Indebetouw, "Three-dimensional microscopy and sectional image reconstruction using optical scanning holography," Appl. Opt. 48, H113-H119 (2009).

18. J. W. Goodman, Introduction to Fourier Optics, 3rd ed. (Roberts, 2005),

19. G. Indebetouw and W. Zhong, "Scanning holographic microscopy of three-dimensional fluorescent specimens," J. Opt. Soc. Am. A 23, 1699-1707 (2006).

20. E. Wolf, "Three-dimensional structure determination of semi-transparent objects from holographic data," Opt. Commun. 1, 153-156 (1969).

21. C. L. Matson and H. Liu, "The Fourier diffraction theorem for turbid media," in "Advances in Optical Imaging and Photon Migration," J. Fujimoto and M. Patterson, eds., Vol. 21 of OSA Trends in Optics and Photonics (Optical Society of America, 1998) p. ATuD14.

22. R. Bracewell, The Fourier Transform and Its Applications, 3rd ed. (McGraw-Hill, 1999),

23. A. J. Devaney, "A computer simulation study of diffraction tomography," IEEE Trans. Biomed. Eng. BME-30, 377-386 (1983).

24. Y. Zou and X. Pan, "Image reconstruction on PI-lines by use of filtered backprojection in helical cone-beam CT," Phys. Med. Biol. 49, 2717-2731 (2004).

25. T. Chan and J. Shen, Image Processing and Analysis: Variational, PDE, Wavelet, and Stochastic Methods (SIAM, 2005).

26. E. Candès, J. Romberg, and T. Tao, "Robust uncertainty principles: Exact signal reconstruction from highly incomplete frequency information," IEEE Trans. Inf. Theory 52, 489-509 (2006).

27. R. Gonzalez and R. Woods, Digital Image Processing, 3rd ed. (Pearson Prentice Hall, 2008).

28. Y. Li and F. Santosa, "A computational algorithm for minimizing total variation in image restoration," IEEE Trans. Image Process. 5, 987-995 (1996).

29. D. Strong and T. Chan, "Edge-preserving and scale- 
dependent properties of total variation regularization," Inverse Probl. 19, S165-S187 (2003).

30. M. K. Ng, H. Shen, E. Lam, and L. Zhang, "A total variation regularization based super-resolution reconstruction algorithm for digital video," EURASIP Journal on Advances in Signal Processing 2007, 1-16 (2007). Article ID 74585.

31. E. Candès and T. Tao, "Near-optimal signal recovery from random projections: Universal encoding strategies?" IEEE Trans. Inf. Theory 52, 5406-5425 (2006).

32. E. Candès and M. B. Wakin, "An introduction to compressive sampling," IEEE Signal Process. Mag. 25, 21-30 (2008).

33. X. Zhang, E. Y. Lam, and T.-C. Poon, "Fast iterative sec- tional image reconstruction in optical scanning holography," in Digital Holography and Three-Dimensional Imaging $(\mathrm{DH})$, OSA Technical Digest (CD) (Optical Society of America, 2009), paper DMA3.

34. S. Boyd and L. Vandenberghe, Convex Optimization (Cambridge Univ. Press, Cambridge, 2004).

35. G. Indebetouw, "A posteriori quasi-sectioning of the threedimensional reconstructions of scanning holographic microscopy,” J. Opt. Soc. Am. A 23, 2657-2661 (2006).

36. X. Zhang, E. Y. Lam, T. Kim, Y. S. Kim, and T.-C. Poon, "Blind sectional image reconstruction for optical scanning holography," Opt. Lett. 34, 3098-3100 (2009). 